\title{
On a magnetic characterization of spectral minimal partitions
}

Received June 24, 2011 and in revised form April 17, 2012

\begin{abstract}
Given a bounded open set $\Omega$ in $\mathbb{R}^{n}$ (or in a Riemannian manifold) and a partition of $\Omega$ into $k$ open sets $D_{j}$, we consider the quantity $\max _{j} \lambda\left(D_{j}\right)$ where $\lambda\left(D_{j}\right)$ is the ground state energy of the Dirichlet realization of the Laplacian in $D_{j}$. If we denote by $\mathfrak{L}_{k}(\Omega)$ the infimum over all the $k$-partitions of $\max _{j} \lambda\left(D_{j}\right)$, a minimal $k$-partition is then a partition which realizes the infimum. When $k=2$, we find the two nodal domains of a second eigenfunction, but the analysis of higher $k$ 's is non-trivial and quite interesting. In this paper, we give the proof of one conjecture formulated in [5] and [16] about a magnetic characterization of the minimal partitions when $n=2$.
\end{abstract}

Keywords. Minimal partitions, nodal sets, Aharonov-Bohm Hamiltonians, Courant's nodal theorem

\section{Introduction}

\subsection{Main definitions}

We mainly consider the Dirichlet Laplacian in a bounded domain $\Omega \subset \mathbb{R}^{2}$. We would like to analyze the relations between the nodal domains of the real-valued eigenfunctions of this Laplacian and the partitions of $\Omega$ into $k$ open sets $D_{i}$ which are minimal in the sense that the maximum over the $D_{i}$ 's of the ground state energy ${ }^{1}$ of the Dirichlet realization of the Laplacian $H\left(D_{i}\right)$ in $D_{i}$ is minimal. In the case of a Riemannian compact manifold, the natural extension is to consider the Laplace-Beltrami operator. We denote by $\lambda_{j}(\Omega)$ the increasing sequence of its eigenvalues and by $u_{j}$ some associated orthonormal basis of real-valued eigenfunctions. The ground state $u_{1}$ can be chosen to be strictly positive in $\Omega$, but the other eigenfunctions $u_{k}$ must have zero sets. For any real-valued $u \in C_{0}^{0}(\bar{\Omega})$, we define the zero set of $u$ as

$$
N(u)=\overline{\{x \in \Omega \mid u(x)=0\}}
$$

and call the components of $\Omega \backslash N(u)$ the nodal domains of $u$. The number of nodal

B. Helffer: Département de Mathématiques, Université Paris-Sud, 91405 Orsay Cedex, France; e-mail: Bernard.Helffer@math.u-psud.fr

T. Hoffmann-Ostenhof: Institut für Theoretische Chemie, Universität Wien, 1090 Wien, Austria; e-mail: thoffmann@tbi.univie.ac.at

Mathematics Subject Classification (2010): 35B05

1 The ground state energy is the smallest eigenvalue. 
domains of $u$ is denoted $\mu(u)$. These $\mu(u)$ nodal domains define a $k$-partition of $\Omega$, with $k=\mu(u)$.

We recall that the Courant nodal theorem says that, for $k \geq 1$, if $\lambda_{k}$ denotes the $k$-th eigenvalue and $E\left(\lambda_{k}\right)$ the eigenspace of $H(\Omega)$ associated with $\lambda_{k}$, then, for all real-valued $u \in E\left(\lambda_{k}\right) \backslash\{0\}, \mu(u) \leq k$.

In dimension 1 the Sturm-Liouville theory says that we always have equality (for Dirichlet in a bounded interval) in the previous theorem (this is what we will call later a Courant-sharp situation). A 1956 theorem due to Pleijel [24] says that this cannot be true when the dimension is larger than one (here we consider the $2 D$-case).

We now introduce, for $k \in \mathbb{N}(k \geq 1)$, the notion of $k$-partition. We define a $k$-partition of $\Omega$ to be a family $\mathcal{D}=\left\{D_{i}\right\}_{i=1}^{k}$ of mutually disjoint sets in $\Omega$. We call it open if the $D_{i}$ are open sets in $\Omega$, and connected if the $D_{i}$ are connected. We denote by $\mathfrak{O}_{k}(\Omega)$ the set of open connected partitions of $\Omega$. We now introduce the notion of spectral minimal partition.

Definition 1.1. For any integer $k \geq 1$, and for $\mathcal{D}$ in $\mathfrak{O}_{k}(\Omega)$, we introduce

$$
\Lambda(\mathcal{D})=\max _{i} \lambda\left(D_{i}\right)
$$

Then we define

$$
\mathfrak{L}_{k}(\Omega)=\inf _{\mathcal{D} \in \mathfrak{O}_{k}} \Lambda(\mathcal{D})
$$

and call $\mathcal{D} \in \mathfrak{O}_{k}$ a minimal k-partition if $\mathfrak{L}_{k}(\Omega)=\Lambda(\mathcal{D})$.

If $k=2$, it is rather well known (see [19] or [13]) that $\mathfrak{L}_{2}(\Omega)=\lambda_{2}(\Omega)$ and that the associated minimal 2-partition is a nodal partition, i.e. a partition whose elements are the nodal domains of some eigenfunction corresponding to $\lambda_{2}$.

A partition $\mathcal{D}=\left\{D_{i}\right\}_{i=1}^{k}$ of $\Omega$ in $\mathfrak{O}_{k}$ is called strong if

$$
\operatorname{Int}\left(\overline{\bigcup_{i} D_{i}}\right) \backslash \partial \Omega=\Omega
$$

where, for a set $A \subset \mathbb{R}^{2}, \operatorname{Int}(A)$ means the interior of $A$.

To a strong partition, we associate a closed set in $\bar{\Omega}$, which is called the boundary set of the partition:

$$
N(\mathcal{D})=\overline{\bigcup_{i}\left(\partial D_{i} \cap \Omega\right)}
$$

$N(\mathcal{D})$ plays the role of the nodal set (in the case of a nodal partition).

This suggests the following definition:

Definition 1.2. We call a partition $\mathcal{D}$ regular if its associated boundary set $N(\mathcal{D})$ has the following properties:

(i) Except for finitely many distinct $x_{i} \in \Omega \cap N$ in the neighborhood of which $N$ is the union of $v_{i}=v\left(x_{i}\right)$ smooth curves $\left(v_{i} \geq 3\right)$ with one end at $x_{i}, N$ is locally diffeomorphic to a regular curve.

(ii) $\partial \Omega \cap N$ consists of a (possibly empty) finite set of points $z_{i}$. Moreover $N$ is near $z_{i}$ the union of $\rho_{i}$ distinct smooth half-curves which hit $z_{i}$.

(iii) $N$ has the equal angle meeting property. 
The $x_{i}$ are called the critical points and form the set $X(N)$. Similarly we denote by $Y(N)$ the set of the boundary points $z_{i}$. By the equal angle meeting property, we mean that the half-curves meet with the some angle at each critical point of $N$ and also at the boundary together with the tangent to the boundary.

We say that $D_{i}, D_{j}$ are neighbors, written $D_{i} \sim D_{j}$, if $D_{i j}:=\operatorname{Int}\left(\overline{D_{i} \cup D_{j}}\right) \backslash \partial \Omega$ is connected. We associate with each $\mathcal{D}$ a graph $G(\mathcal{D})$ by associating with each $D_{i}$ a vertex and to each pair $D_{i} \sim D_{j}$ an edge. We will say that the graph is bipartite if it can be colored by two colors (two neighbors having two different colors). We recall that the graph associated with a collection of nodal domains of an eigenfunction is always bipartite.

\subsection{Motivation and overview}

Before we state some results on spectral minimal partitions, discuss their properties and finally formulate and prove the central result of the present paper, we give an informal overview of our results. The main result is a new characterization of minimal partitions via specific magnetic Hamiltonians; see Section 4 for the necessary definitions and explanations of those operators.

In [21] we have characterized via minimal partitions the case of equality in Courant's nodal theorem (see Theorem 2.3 below). Roughly speaking (see Theorem 2.2), if a minimal partition could in principle stem from an eigenfunction it must be already produced by the nodal domains of an eigenfunction, and this can only happen if there is equality in (7). Pleijel's result of [24] implies, roughly speaking, that eigenfunctions associated to higher eigenvalues cannot lead to equality in (7).

In Section 3 we give a few pictures of non-nodal minimal partitions, or more precisely natural candidates, since it is notoriously hard to work out explicit examples for such partitions. A first glance shows that there are points where an odd number of nodal arcs meet.

More than 10 years ago together with Maria Hoffmann-Ostenhof and Mark Owen we investigated some special magnetic Schrödinger operators, called Aharonov-Bohm Hamiltonians, i.e. Hamiltonians with zero magnetic field but with singular magnetic vector potential and with half-integer circulation around holes [17, 18] (see Section 4). This investigation was motivated by the result of Berger and Rubinstein [3], surprising at that time, about the zero set of a groundstate for such a problem with one hole. For more than one hole similar results were obtained on zero sets: each hole was hit by an odd number of nodal arcs. $^{2}$

The findings in [17, 18] motivated the conjecture in [5] and [16], reformulated and proved in the present paper. The result says roughly that spectral minimal partitions are obtained by minimizing a certain eigenvalue of an Aharonov-Bohm Hamiltonian with respect to the number and the position of poles if we assume that $\Omega$ is simply connected. See Theorem 5.1 for the full result.

This new approach to spectral minimal partitions sheds new light on them. While in the original formulation [21], say for a fixed $\Omega$, the $\mathfrak{L}_{k}(\Omega)$ and the associated minimal

\footnotetext{
2 Similar results for punctured domains were later obtained in [1].
} 
partitions as defined in Definition 1.1 require the calculation of $\Lambda(\mathcal{D})$ for $k$-partitions, the new formulation can be considered as an, admittedly involved, eigenvalue minimization.

\section{Basic properties of minimal partitions}

The following theorems have been proved by Conti-Terracini-Verzini $[11,12,13]$ and Helffer-T. Hoffmann-Ostenhof-Terracini [21]:

Theorem 2.1. For any $k$, there exists a minimal regular $k$-partition. Moreover any minimal $k$-partition has a regular representative. ${ }^{3}$

Other proofs of a somewhat weaker version of this statement have been given by BucurButtazzo-Henrot [8] and Caffarelli-F. H. Lin [10].

A natural question is whether a minimal partition of $\Omega$ is a nodal partition, i.e. the family of nodal domains of an eigenfunction of $H(\Omega)$. We have first the following converse theorem ([19], [21]):

Theorem 2.2. If the graph of a minimal partition is bipartite, then this partition is nodal.

A natural question is now to determine how general the previous situation is. Surprisingly this only occurs in the so called Courant-sharp situation. We say that $u$ is Courant-sharp if

$$
u \in E\left(\lambda_{k}\right) \backslash\{0\} \quad \text { and } \quad \mu(u)=k .
$$

For any integer $k \geq 1$, we denote by $L_{k}(\Omega)$ the smallest eigenvalue of $H(\Omega)$ whose eigenspace contains an eigenfunction with $k$ nodal domains. We set $L_{k}(\Omega)=\infty$ if there are no such eigenfunctions. In general, one can show that

$$
\lambda_{k}(\Omega) \leq \mathfrak{L}_{k}(\Omega) \leq L_{k}(\Omega) .
$$

The last result gives the full picture of the equality cases:

Theorem 2.3. Suppose $\Omega \subset \mathbb{R}^{2}$ is regular. If $\mathfrak{L}_{k}(\Omega)=L_{k}(\Omega)$ or $\mathfrak{L}_{k}(\Omega)=\lambda_{k}(\Omega)$ then

$$
\lambda_{k}(\Omega)=\mathfrak{L}_{k}(\Omega)=L_{k}(\Omega) .
$$

In addition, one can find in $E\left(\lambda_{k}\right)$ a Courant-sharp eigenfunction.

This answered a question posed in [9, Section 7].

Remark 2.4. Very recently spectral partitions for discrete problems, namely quantum graphs, have been investigated in [2].

\section{Examples of minimal $k$-partitions for special domains}

Using Theorem 2.3, it is now easier to analyze the situation for the disk or for rectangles (at least in the irrational case), since we have just to check for which eigenvalues one can find associated Courant-sharp eigenfunctions.

\footnotetext{
3 Modulo sets of capacity 0.
} 
The possible topological types of a minimal partition $\mathcal{D}$ rely essentially on Euler's formula and the fact that the $D_{i}$ 's have to be nice, in the sense that

$$
\operatorname{Int}\left(\bar{D}_{i}\right) \cap \Omega=D_{i}
$$

Figures 2 and 3 illustrate possible situations.

Proposition 3.1. Let $U$ be an open set in $\mathbb{R}^{2}$ with piecewise- $C^{1}$ boundary and let $N$ be a closed set such that $U \backslash N$ has $k$ components and that $N$ has the properties of Definition 1.2. Let $b_{0}$ be the number of components of $\partial U$ and $b_{1}$ be the number of components of $N \cup \partial U$. Denote by $v\left(x_{i}\right)$ and $\rho\left(z_{i}\right)$ the numbers of arcs associated with the $x_{i} \in X(N)$, respectively $z_{i} \in Y(N)$. Then

$$
k=b_{1}-b_{0}+\sum_{x_{i} \in X(N)}\left(\frac{v\left(x_{i}\right)}{2}-1\right)+\frac{1}{2} \sum_{z_{i} \in Y(N)} \rho\left(z_{i}\right)+1
$$

This allows us to analyze minimal partitions of a specific topological type. If in addition the domain has some symmetries and we assume that a minimal partition keeps some of these symmetries, then we find natural candidates for minimal partitions.

\section{Minimal 3-partitions}

In the case of the disk (see [20]), we have no proof that the minimal 3-partition is the "Mercedes star" or $Y$-partition, i.e. the partition created by three straight rays meeting at the center with equal angles. But if we assume that the minimal 3-partition has a unique singular point at the center then one can show it is indeed the $Y$-partition. This point of view is explored numerically by Bonnaillie-Helffer [5] (using some method equivalent to the Aharonov-Bohm approach and playing with the location of the critical point). There is also an interesting theoretical analysis by Noris-Terracini [23].

We have no example of minimal 3-partitions with two critical points. For the disk and the square the minimal 4-partitions are nodal.

\section{Minimal 5-partitions}

Using the covering approach, we were able (with V. Bonnaillie) in [5] to produce numerically the following candidate $\mathcal{D}_{1}$ for a minimal 5-partition assuming a specific topological type.

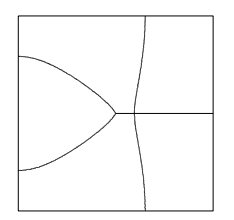

Fig. 1. Candidate $\mathcal{D}_{1}$ for the 5 -partition of the square. 
It is interesting to compare it with other possible topological types of minimal 5-partitions. They can be classified by using Euler's formula (see formula (9)). Inspired by numerical computations in [14], one looks for a configuration which has the symmetries of the square and four critical points. We get two types of models that we can reduce to a Dirichlet-Neumann problem on a triangle corresponding to the eighth of the square. Moving the Neumann boundary on one side as in [7] leads to two candidates $\mathcal{D}_{2}$ and $\mathcal{D}_{3}$. One has lower energy $\Lambda(\mathcal{D})$ and one recovers the pictures in [14].
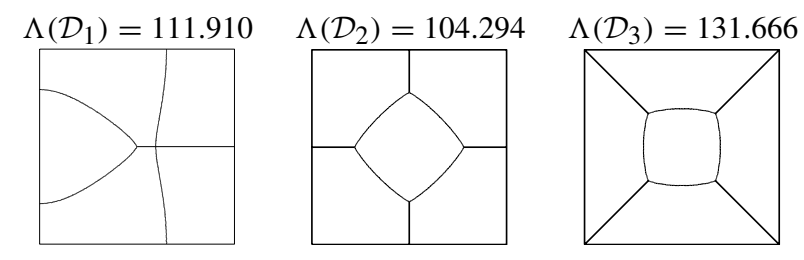

Fig. 2. Three candidates for the 5-partition of the square.

Note that in the case of the disk a similar analysis leads to a different answer. The partition of the disk by five half-rays with equal angles has lower energy than the minimal 5-partition with four singular points.

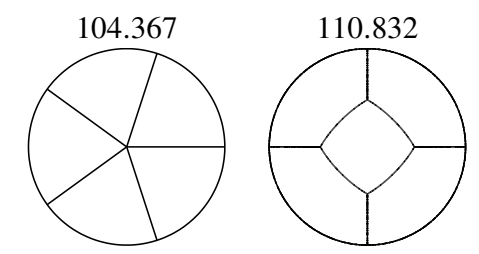

Fig. 3. Two candidates for the 5-partition of the disk.

\section{The Aharonov-Bohm approach}

Let us recall some definitions and results about the Aharonov-Bohm Hamiltonian (for short $\mathbf{A B} X$-Hamiltonian) defined in an open set $\Omega$ which can be simply connected or not. These results were initially motivated by the work of Berger-Rubinstein [3], and further developed in $[1,17,18,6,5]$.

\section{Simply connected case: one pole}

We first consider the case when one pole, denoted by $X=\left(x_{0}, y_{0}\right)$, is chosen in $\Omega$ and introduce the magnetic potential

$$
A^{X}(x, y)=\left(A_{1}^{X}(x, y), A_{2}^{X}(x, y)\right)=\frac{\Phi}{2 \pi}\left(-\frac{y-y_{0}}{r^{2}}, \frac{x-x_{0}}{r^{2}}\right) .
$$


We know that in this case the magnetic field vanishes identically in

$$
\dot{\Omega}_{X}=\Omega \backslash\{X\} .
$$

The $\mathbf{A B} X$-Hamiltonian is defined by considering the Friedrichs extension starting from $C_{0}^{\infty}\left(\dot{\Omega}_{X}\right)$, and the associated differential operator is

$$
-\Delta_{\mathbf{A}^{X}}:=\left(D_{x}-A_{1}^{X}\right)^{2}+\left(D_{y}-A_{2}^{X}\right)^{2} \quad \text { with } \quad D_{x}=-i \partial_{x} \text { and } D_{y}=-i \partial_{y} .
$$

We will consider in what follows the very special case when the flux $\Phi$ created at $X=$ $\left(x_{0}, y_{0}\right)$, which can be computed by considering the circulation of $\mathbf{A}^{X}$ along a simple closed path turning once anti-clockwise around $X$, satisfies

$$
\frac{\Phi}{2 \pi}=\frac{1}{2} \text {. }
$$

Under assumption (13), let $K_{X}$ be the anti-linear operator

$$
K_{X}=e^{i \theta_{X}} \Gamma,
$$

with $\left(x-x_{0}\right)+i\left(y-y_{0}\right)=\sqrt{\left|x-x_{0}\right|^{2}+\left|y-y_{0}\right|^{2}} e^{i \theta_{X}}$, where $\Gamma$ is the complex conjugation operator $\Gamma u=\bar{u}$ and

$$
\nabla \theta_{X}=2 \mathbf{A}^{X}
$$

which can also be rewritten in the form

$$
-\mathbf{A}^{X}=\mathbf{A}^{X}-\nabla \theta_{X}
$$

The flux condition (13) shows that one can find a solution $\theta_{X}$ of (14) (a priori multivalued) such that $e^{i \theta_{X}}$ is single-valued and $C^{\infty}$. Hence $-\Delta_{\mathbf{A}^{X}}$ and $-\Delta_{-\mathbf{A}^{X}}$ are intertwined by the gauge transformation associated with $e^{i \theta_{X}}$. Then we have

$$
K_{X} \Delta_{\mathbf{A}^{X}}=\Delta_{\mathbf{A}^{X}} K_{X}
$$

We say that a function $u$ is $K_{X}$-real if it satisfies $K_{X} u=u$. Then the operator $-\Delta_{\mathbf{A}^{X}}$ preserves the $K_{X}$-real functions. In the same way one proves that the usual Dirichlet Laplacian admits an orthonormal basis of real-valued eigenfunctions or one restricts this Laplacian to the vector space over $\mathbb{R}$ of real-valued $L^{2}$ functions, one can construct for $-\Delta_{\mathbf{A}^{X}}$ a basis of $K_{X}$-real eigenfunctions or, alternatively, consider the restriction of the AB $X$-Hamiltonian to the vector space over $\mathbb{R}$

$$
L_{K_{X}}^{2}\left(\dot{\Omega}_{X}\right)=\left\{u \in L^{2}\left(\dot{\Omega}_{X}\right): K_{X} u=u\right\} .
$$

\section{Non-simply connected case}

In this situation, magnetic potentials in $\Omega$ with zero magnetic field can be different from gradients if some fluxes around some holes are not in $2 \pi \mathbb{Z}$. In this situation we will be interested in potentials where the flux created by some hole is $\pi$. This will be realized in this article by introducing a pole in the hole. Except that $\dot{\Omega}_{X}=\Omega$ (there is no singularity in $\Omega$ ) all what has been defined before goes through and this is actually the initial case treated in the pioneering work [3]. 


\section{Poles and holes}

We can extend our construction of an Aharonov-Bohm Hamiltonian in the case of a configuration with $\ell$ distinct points $X_{1}, \ldots, X_{\ell}$ (putting flux $\pi$ at each of these points). These points can be chosen in $\Omega$ or in the holes. They are distinct and each hole contains at most one $X_{k}$. We can just take as magnetic potential

$$
\mathbf{A}^{\mathbf{X}}=\sum_{j=1}^{\ell} \mathbf{A}^{X_{j}}
$$

where $\mathbf{X}=\left(X_{1}, \ldots, X_{\ell}\right)$. Our Hamiltonian will be defined in $\dot{\Omega}_{\mathbf{X}}=\Omega \backslash \mathbf{X}$. We can also construct (see $[17,18]$ ) the anti-linear operator $K_{\mathbf{X}}$, where $\theta_{X}$ is replaced by a multivalued function $\phi_{\mathbf{X}}$ such that $\nabla \phi_{\mathbf{X}}=2 \mathbf{A}^{\mathbf{X}}$ and $e^{i \phi_{\mathbf{X}}}$ is single-valued and $C^{\infty}$. We can then consider the real subspace of $K_{\mathbf{X}}$-real functions in $L_{K_{\mathbf{X}}}^{2}\left(\dot{\Omega}_{\mathbf{X}}\right)$ and our operator as an unbounded selfadjoint operator on $L_{K_{\mathbf{X}}}^{2}\left(\dot{\Omega}_{\mathbf{X}}\right)$.

It was shown in $[17,18]$ for the case with holes and in [1] for the case with poles that the nodal set of such a $K_{\mathbf{X}}$-real eigenfunction has the same structure as the nodal set of a real-valued eigenfunction of the Laplacian except that an odd number of half-lines meet at each pole and at the boundary of each hole containing some $X_{k}$. In the case of one hole, this fact was first observed by Berger-Rubinstein [3] for the first eigenfunction (assuming that the first eigenvalue is simple). We denote by $L_{k}\left(\dot{\Omega}_{\mathbf{X}}\right)$ the lowest eigenvalue, if it exists, such that there exists a $K_{\mathbf{X}}$-real eigenfunction with $k$ nodal domains and we set $L_{k}\left(\dot{\Omega}_{\mathbf{X}}\right)=+\infty$ if there is no such eigenvalue.

\section{The magnetic characterization of a minimal partition}

We now prove the following conjecture presented (in the simply-connected case) in [5] and [16].

Theorem 5.1. Suppose $\Omega$ is a bounded, not necessarily simply connected, domain with $m$ disjoint closed holes $B_{i}(i=1, \ldots, m)$ with non-empty interiors. Again assume that $\partial \Omega$ is piecewise $C^{1}$. Then

$$
\mathfrak{L}_{k}(\Omega)=\inf _{\ell \in \mathbb{N}} \inf _{X_{1}, \ldots, X_{\ell}} L_{k}\left(\dot{\Omega}_{\mathbf{X}}\right)
$$

where in the infimum each $X_{j}=\left(x_{j}, y_{j}\right)$ is either in $\operatorname{Int}\left(B_{i}\right)$ or in $\Omega$. In each $B_{i}$ there is either one or no $X_{i}$. The $X_{i} \in \Omega$ are distinct points.

Let us first give the proof in the simply connected case.

Step 1: $\inf _{\ell \in \mathbb{N}} \inf _{X_{1}, \ldots, X_{\ell}} L_{k}\left(\dot{\Omega}_{\mathbf{X}}\right) \leq \mathfrak{L}_{k}(\Omega)$. Considering a minimal $k$-partition $\mathcal{D}=$ $\left(D_{1}, \ldots, D_{k}\right)$, we know that it has a regular representative and we denote by $X^{\text {odd }}(\mathcal{D}):=$ $\left(X_{1}, \ldots, X_{\ell}\right)$ the critical points of the boundary set of the partition for which an odd number of half-curves meet. 
To prove Step 1, we have indeed just to prove that, for this family of points $\mathbf{X}=$ $X^{\text {odd }}(\mathcal{D}), \mathfrak{L}_{k}(\Omega)$ is an eigenvalue of the Aharonov-Bohm Hamiltonian associated with $\dot{\Omega}_{\mathbf{X}}$ and to explicitly construct the corresponding eigenfunction with $k$ nodal domains described by the $D_{i}$ 's.

For this, we recall that we have proven in [21] the existence of a family $\left(u_{i}\right)_{i=1}^{k}$ such that $u_{i}$ is a ground state of $H\left(D_{i}\right)$ and $u_{i}-u_{j}$ is a second eigenfunction of $H\left(D_{i j}\right)$ when $D_{i} \sim D_{j}$. The claim is that one can find a sequence $\epsilon_{i}(x)$ of $\mathbb{S}^{1}$-valued functions, where $\epsilon_{i}$ is a suitable ${ }^{4}$ square root of $e^{i \phi_{\mathbf{X}}}$ in $D_{i}$, such that $\sum_{i} \epsilon_{i}(x) u_{i}(x)$ is an eigenfunction of the ABX-Hamiltonian associated with the eigenvalue $\mathfrak{L}_{k}$.

More explicitly, let us describe how we can construct $\epsilon_{i}(x)$. We start from some $i_{0}$ and define $\epsilon_{i_{0}}(x)=e^{(i / 2) \phi_{\mathbf{X}}}$. According to the footnote, $\epsilon_{i_{0}}(x)$ is a well defined $C^{\infty}$ function. Let $D_{i}$ a nearest neighbor of $D_{i_{0}}$. Then we define $\epsilon_{i}(x)=-e^{(i / 2) \phi_{\mathbf{x}}}$. Then we can extend the definition by considering the neighbors of the neighbors. We have to check that the construction is consistent. The problem can be reduced to the following question. Consider a closed simple path $\gamma$ in $\dot{\Omega}_{\mathbf{X}}$ transversal to $\mathcal{N}(\mathcal{D})$ (and avoiding the critical points). Take some origin $x_{0}$ on $\gamma \cap D_{i_{1}}$. We start from $\epsilon(x)=e^{(i / 2) \phi_{\mathbf{X}}(x)}$ in $D_{i_{1}}$ and, choosing the positive orientation, multiply by -1 each time we cross an $\operatorname{arc}$ of $\mathcal{N}(\mathcal{D})$. It is then a consequence of Euler's formula that the number of crossings along $\gamma$ is odd if and only if there are an odd number of points of $\mathbf{X}$ inside $\gamma$ (apply Euler's formula (9) with $U$ being the open set bounded by $\gamma)$. It is then clear that $\epsilon(x)$ is well defined along $\gamma$.

Step 2: $\inf _{\ell \in \mathbb{N}} \inf _{X_{1}, \ldots, X_{\ell}} L_{k}\left(\dot{\Omega}_{\mathbf{X}}\right) \geq \mathfrak{L}_{k}(\Omega)$. Conversely, given $\ell$ distinct points $X_{i}$ in $\Omega$, any family of nodal domains of a $K_{\mathbf{X}}$-real eigenfunction of the Aharonov-Bohm operator on $\dot{\Omega}_{\mathbf{X}}$ corresponding to $L_{k}$ gives a $k$-partition. Using the results of [17] and [1], we immediately see that the $X_{i}$ 's correspond to the "odd" singular points of the partitions. In each of these nodal domains $D_{i}, L_{k}$ is an eigenvalue of the Dirichlet realization of the Schrödinger operator with magnetic potential $\mathbf{A}^{\mathbf{X}}$, which is by the diamagnetic inequality higher than the ground state energy of the Dirichlet Laplacian in $D_{i}$ without magnetic field. Hence the energy $\Lambda_{k}(\mathcal{D})$ of this partition is indeed less than $L_{k}\left(\dot{\Omega}_{\mathbf{X}}\right)$.

Step 3: Proof in the non-simply connected case. The main change is in Step 1. In the non-simply connected case, the set $\mathbf{X}$ consists of the singular points of the boundary set inside $\Omega$ where an odd number of half-lines arrive together with those points in the holes whose boundary is hit by an odd number of half-curves.

Examples. Let us present a few examples illustrating the theorem in the case of a simply connected domain. When $k=2$, there is no need to consider punctured $\Omega$ 's. The infimum is obtained for $\ell=0$. When $k=3$, it is possible to show (see Remark 5.3 below) that it is enough to minimize over $\ell=0, \ell=1$ and $\ell=2$. In the case of the disk and the square, it is proven that the infimum cannot occur for $\ell=0$ and we conjecture that the infimum is for $\ell=1$ and is attained for the punctured domain at the center. For $k=5$, it seems that the infimum is for $\ell=4$ in the case of the square (see Figure 2) and for $\ell=1$ in the case of the disk (see Figure 3).

\footnotetext{
4 Note that by construction the $D_{i}$ 's never contain any point of $\mathbf{X}$. Hence the ground state energy of the Hamiltonian $H\left(D_{i}\right)$ is the same as the ground state energy of $H_{\mathbf{A}} \mathbf{x}\left(D_{i}\right)$.
} 
Remark 5.2. If $\mathcal{D}$ is a regular representative of a minimal $k$-partition and if $\dot{\Omega}_{\mathbf{X}}$ is constructed as in Step 1 of the above proof, then $\mathfrak{L}_{k}(\Omega)=\lambda_{k}\left(\dot{\Omega}_{\mathbf{X}}\right)$ (Courant -sharp situation). Coming back indeed to this step, one can follow the proof of Theorem 1.13 (Section 6) in [21].

Remark 5.3. Euler's formula (9) implies that for a minimal $k$-partition $\mathcal{D}$ of a simply connected domain $\Omega$ the cardinality of $X^{\text {odd }}(\mathcal{D})$ satisfies

$$
\# X^{\text {odd }}(\mathcal{D}) \leq 2 k-3 \text {. }
$$

Note that if $b_{1}=b_{0}$, we necessarily have a singular point on the boundary. The argument depends only on Euler's formula. If we implement the additional property that the open sets $D_{i}$ of a minimal partition are nice (see (8)), we can exclude the case when there is only one point on the boundary. We emphasize that this was not a priori excluded from the results of $[17,1]$. Hence, we obtain

$$
b_{1}-b_{0}+\frac{1}{2} \sum \rho\left(y_{i}\right) \geq 1,
$$

which implies the inequality

$$
\# X^{\text {odd }}(\mathcal{D}) \leq 2 k-4
$$

This estimate seems optimal for a general geometry although all the known candidates for minimal partitions for $k=3$ and 5 have fewer odd critical points.

Remark 5.4. The argument around (8) shows that a nodal set of a $K_{\mathbf{X}}$-real eigenfunction that corresponds to a minimal partition cannot have a critical point that is met only by one nodal arc. Actually that can happen for ground states of Aharonov-Bohm Hamiltonians (see [17]) which of course do not correspond to minimal partitions.

Remark 5.5. It would be interesting to look at the case of the sphere (already considered in [22]) and the first problem in this case is to define the suitable magnetic Laplacian. We refer to [25] for one of the first papers on this question. More specifically, we would like to construct in our case an Aharonov-Bohm Hamiltonian. Note for example that we cannot have such an operator with one pole and flux $\pi$ around this pole. Fortunately there are no minimal $k$-partitions whose boundary set consists of one "odd" critical point on the sphere, as can be seen by Euler's formula for the sphere (see [22, Remark 4.2]). We indeed know that the number of "odd" critical points is even. This is actually a standard result from graph theory that the number of vertices with odd degree is even (see for example Corollary 1.2 in [4]).

This suggests that instead of putting flux $\pi$ around each pole, we take alternately $\pi$ and $-\pi$ for the fluxes in order to get a total flux equal to 0 . In other words, we should probably describe $X^{\text {odd }}(\mathcal{D})$ as a union of dipoles.

Acknowledgments. When writing this paper we benefited from a useful discussion with V. Bonnaillie-Noël and S. Terracini. 


\section{References}

[1] Alziary, B., Fleckinger-Pellé, J., Takáč, P.: Eigenfunctions and Hardy inequalities for a magnetic Schrödinger operator in $\mathbb{R}^{2}$. Math. Methods Appl. Sci. 26, 1093-1136 (2003) Zbl 1045.81023 MR 1994668

[2] Band, R., Berkolaiko, G., Raz, H., Smilansky, U.: The number of nodal domains on quantum graphs as a stability index of graph partitions. Comm. Math. Phys. 311, 815-838 (2012) Zbl 1244.81026 MR 2909765

[3] Berger, J., Rubinstein, J.: On the zero set of the wave function in superconductivity. Comm. Math. Phys. 202, 621-628 (1999) Zbl 0938.82063 MR 1690956

[4] Bondy, J. A., Murty, U. S. R.: Graph Theory. Grad. Texts in Math. 243, Springer (2008) Zbl 1134.05001 MR 2368647

[5] Bonnaillie-Noël, V., Helffer, B.: Numerical analysis of nodal sets for eigenvalues of Aharonov-Bohm Hamiltonians on the square and application to minimal partitions. Exp. Math. 20, 304-322 (2011) Zbl pre06152641 MR 2836255

[6] Bonnaillie-Noël, V., Helffer, B., Hoffmann-Ostenhof, T.: Aharonov-Bohm Hamiltonians: isospectrality and minimal partitions. J. Phys. A 42, no. 18, 185203, 20 pp. (2009)

[7] Bonnaillie-Noël, V., Helffer, B., Vial, G.: Numerical simulations for nodal domains and spectral minimal partitions. ESAIM Control Optim. Calc. Var. 16, 221-246 (2010) Zbl 1191.35189 MR 2598097

[8] Bucur, D., Buttazzo, G., Henrot, A.: Existence results for some optimal partition problems. Adv. Math. Sci. Appl. 8, 571-579 (1998) Zbl 0915.49006 MR 1657219

[9] Burdzy, K., Hołyst, R., Ingerman, D., March, P.: Configurational transition in a FlemingViot-type model and probabilistic interpretation of Laplacian eigenfunctions. J. Phys. A 29, 2633-2642 (1996) Zbl 0901.60054

[10] Caffarelli, L. A., Lin, F. H.: An optimal partition problem for eigenvalues. J. Sci. Comput. 31, 5-18 (2007) Zbl 1123.65060 MR 2304268

[11] Conti, M., Terracini, S., Verzini, G.: An optimal partition problem related to nonlinear eigenvalues. J. Funct. Anal. 198, 160-196 (2003) Zbl 1091.35051 MR 1962357

[12] Conti, M., Terracini, S., Verzini, G.: A variational problem for the spatial segregation of reaction-diffusion systems. Indiana Univ. Math. J. 54, 779-815 (2005) Zbl 1132.35397 MR 2151234

[13] Conti, M., Terracini, S., Verzini, G.: On a class of optimal partition problems related to the Fučik spectrum and to the monotonicity formula. Calc. Var. 22, 45-72 (2005) Zbl 1132.35365 MR 2105968

[14] Cybulski, O., Babin, V., Hołyst, R.: Minimization of the Renyi entropy production in the space-partitioning process. Phys. Rev. E 71, 046130 (2005) MR 2139992

[15] Helffer, B.: On spectral minimal partitions: a survey. Milan J. Math. 78, 575-590 (2010) Zbl 1225.35158 MR 2781853

[16] Helffer, B.: An introduction to some conjectures for spectral minimal partitions. J. Egyptian Math. Soc. 19, 45-51 (2011) MR 2914127

[17] Helffer, B., Hoffmann-Ostenhof, M., Hoffmann-Ostenhof, T., Owen, M. P.: Nodal sets for ground states of Schrödinger operators with zero magnetic field in non-simply connected domains. Comm. Math. Phys. 202, 629-649 (1999) Zbl 1042.81012 MR 1690957

[18] Helffer, B., Hoffmann-Ostenhof, M., Hoffmann-Ostenhof, T., Owen, M. P.: Nodal sets, multiplicity and superconductivity in non-simply connected domains. In: Connectivity and Superconductivity, Lecture Notes in Phys. 62, Springer, 63-86 (2000) Zbl 0986.35104

[19] Helffer, B., Hoffmann-Ostenhof, T.: Converse spectral problems for nodal domains. Moscow Math. J. 7, 67-84 (2007) Zbl 1125.35068 MR 2324557 
[20] Helffer, B., Hoffmann-Ostenhof, T.: On minimal partitions: new properties and applications to the disk. In: Spectrum and Dynamics, CRM Proc. Lecture Notes 52, Amer. Math. Soc., 119-135 (2010) MR 2743435

[21] Helffer, B., Hoffmann-Ostenhof, T., Terracini, S.: Nodal domains and spectral minimal partitions. Ann. Inst. H. Poincaré Anal. Non Linéaire 26, 101-138 (2009) Zbl 1171.35083 MR 2483815

[22] Helffer, B., Hoffmann-Ostenhof, T., Terracini, S.: On spectral minimal partitions: the case of the sphere. In: Around the Research of Vladimir Maz'ya III, Int. Math. Ser. 13, Springer, 153-179 (2010) Zbl 1230.35072 MR 2664708

[23] Noris, B., Terracini, S.: Nodal sets of magnetic Schrödinger operators of Aharonov-Bohm type and energy minimizing partitions. Indiana Univ. Math. J. 59, 1361-1404 (2010) Zbl 1219.35054 MR 2815036

[24] Pleijel, A.: Remarks on Courant's nodal theorem. Comm. Pure Appl. Math. 9, 543-550 (1956) Zbl 0070.32604 MR 0080861

[25] Wu, T. T., Yang, C. N.: Concept of nonintegrable phase factors and global formulation of gauge fields. Phys. Rev. D 12, 3845-3857 (1975) MR 0426712 